\title{
Quality of Life After Bariatric Surgery
}

\author{
Moly K.T. \\ Professor cum Principal, Amrita College of Nursing, Amrita Institute of Medical Sciences, AMRITA Vishwa \\ Vidyapeetham, Kochi, Kerala, India. PIN: 682041
}

\begin{abstract}
Bariatric surgery improves patients' well-being beyond significant weight loss and resolution of comorbidities, Quality of Life (QoL) is a relevant but challenging variable to assess in the process of weight loss and lifestyle changes. The primary objective of the study was to determine the QoL of patients after bariatric surgery in comparison with obese patients who have not undergone bariatric surgery. A descriptive analytical approach was used to collect data from 100 subjects who had undergone Laparoscopic Sleeve Gastrectomy (LSG) through SF 36 in comparison with 100 obese subjects without surgery. 50\% data collection was done directly from OPD and 50\% from telephonic contact in both the groups. The mean scores in all the eightdimensions of SF 36 were found to be good in Bariatric Surgery (BS) group. Both Physical Component Score (PCS) and Mental Component Score (MCS) were found to be high, the MCS being higher $(85.74+$ $22.72)$ than the PCS $(83.68+21.8)$ in BS group whereas the MCS was poor in obese group $(49.98+11.84)$. The overall QoL of BS group was found to be good (mean $80.93+18.18$ ) compared to obese group $(49.69+$ 11.77). The health related QoL was found to be quite good in patients after LSG than those without surgery. The study concludes that the health related quality of life is better in obese patients after LSG than those without surgery. The study also strongly recommends the conduct of long term longitudinal qualitative studies with multiple points of measurements and follow-ups to be extended to the community.
\end{abstract}

Keywords: Quality of Life, Laparoscopic Sleeve Gastrectomy, Physical Component Score, Mental Component Score, Obesity.

\section{Introduction}

LSG has gained grounds worldwide as the popular bariatric surgical procedure including Asia. Sleeve gastrectomy has been found to improve Quality of Life (QoL) and allows reduction of comorbidities. ${ }^{1,2,3}$ Beyond significant weight loss, the objective of bariatric surgery is to improve patients' postoperative well-being. QoL is recognized as a relevant variable to consider in the weight loss process and psychologic monitoring of patients.

\section{Corresponding Author:}

\section{Moly K.T.}

Professor cum Principal, Amrita College of Nursing, Amrita Institute of Medical Sciences, AMRITA Vishwa Vidyapeetham, Kochi, Kerala, India. PIN: 682041

Ph: No. +9194475 13383

e-mail: ktmoly59@gmail.com
It has to be remembered that almost all the patients who had undergone obesity surgery,were not successful in following or maintaining a prescribed diet in the past. Adherence to the dietary restrictions and altered lifestyle necessary after LSG requires continuous monitoring and support from relatives and caregivers. ${ }^{4}$

The importance of optimizing patients' monitoring, notably around 15 to 18 months after bariatric surgery (the first "critical" period of weight regain) especially for men and diminished self-perceived QoL especially for women has been highlighted in literature. ${ }^{5}$

QoL is a multidimensional construct. When applied to obesity, it includes not only health-related and weightrelated QoL, but also the psychosocial burden. These relationships stress the necessity of its assessment. ${ }^{6}$ But less is known about its psychosocial impact. Depressive disorders are the most common psychological diagnoses (ranging from $4.4 \%$ to $53 \%$ ), followed by anxiety disorder. ${ }^{7,8}$ In a study on Quality of Life and Depression 
in obese conducted among 220 patients attending various OPDs of a tertiary care hospital, the QoL in mental components $(49.43+/-13.68)$ was found to be poor than the physical component $(54.4+/-20.09){ }^{9}$

The long term complications after bariatric surgery cannot be neglected. The chronic complications include stricture (3.5\%), nutritional deficiencies, Vit. $\mathrm{B}_{12}$, Vit. $\mathrm{D}$, folate, iron \& zinc deficiency $(3 \%, 23 \%, 3 \%, 3 \%$ \& $14 \%$ respectively) and gastrooesophageal reflex disease [GERD] (47\%). ${ }^{10}$

Although there seems to be adequate post surgical physical care following BS there appears to be a lack of postsurgical psychosocial follow up and support for these patients. ${ }^{11}$ The psychological distress and the vulnerability in social situations while adjustingto the new rapidly changing body image after LSG needs attention.

Massive weight loss often leaves the patient with large quantities of flabby skin that can result in problems related to altered body image. Additional surgeries may be required to handle the excess skin which may cause confusion about the impact of bariatric surgery.

Most of the studies conducted in India were to compare the effect of different types of bariatric surgical procedures in terms of weight loss and resolution of comorbidities. Studies in India on QoL after LSG were found to be less. Also it is interesting to note that women account for $80 \%$ of those having BS, although the reasons are poorly understood. ${ }^{12}$

Successful QoL after bariatric surgery depends on proper selection of patients and a thorough pre operative preparation by a multidisciplinary health care team. The nurses' role in making the patient and family members clear about the real expectations as well as their own responsibilities in the diet and life style on a long term basis is to be understood.

The purpose of the study was to explore the health related QoL in patients after bariatric surgery (Laparoscopic Sleeve Gastrectomy) in comparison with obese patients without surgery so as to provide the right perception of the surgical outcome to the candidates for bariatric surgery as well as the health care team along with an awareness of each one's responsibilities towards attaining the best possible QoL.

The primary objective of the study was to determine the QoL of patients after bariatric surgery in comparison with obese patients who have not undergone bariatric surgery. Accordingly, the null hypothesis tested was"There will be no significant difference between the mean Quality of Life score of obese patients after bariatric surgery (BS group) and obese patients who did not undergo bariatric surgery (Obese group/Control group)"

\section{Materials and Method}

In view of the nature of problems selected for the study and objectives, a descriptive analytical approach was used. The study sample included 100 subjects who had undergone bariatric surgery six months before and 100 obese subjects without surgery at the same time meeting the selection criteria for bariatric surgery as per ASMBS 2012. As subjects after bariatric surgery rarely need to come to OPD after six months, 50\% data collection was done from GI Surgery OPD and 50\% from telephonic contact. Data collection from obese patients was also done in the same way ie, 50\% from OPDs (Medicine, Endocrinology OPDs and Obesity Clinic) and $50 \%$ through telephonic contact. Data collection took around one year. QoL was assessed through RAND SF 36 Item Health Survey 1.0. ${ }^{13}$ The item-and scale-level statistical analyses supported the validity and reliability of SF-36 for use in India. ${ }^{14}$

\section{Results}

Sample Characteristics: The group of patients after bariatric surgery was referred as 'BS group' and obese patients who have not undergone surgery were referred as 'Obese group' for easy understanding. The study subjects in BS group were comparatively of younger age than the obese group. (76\% in BS group belonged to the age group 31-55 years whereas in obese group it was only $65 \%$ ) Majority of the subjects in both the groups were females (BS group - $61 \%$ and obese group - $73 \%$ ). The BS group was more educated than the obese group (58\% of subjects in BS groups were graduates or post graduates whereas it was only $28 \%$ in obese).

Nearly half of study subjects in BS group (46\%) changed to the category of overweight after surgery. However, $4 \%$ in BS group were still in class 3 obesity, $14 \%$ in class 2 obesity and $36 \%$ in class 1 obesity category. The mean weight before surgery $115.99+22.97$ was reduced to a mean of $84.02+17.75$ after surgery. The mean percentage of EWL was $63.31+26.20$. The mean weight of BS group was $84.02+17.75$ at the time of 
study while the mean weight of obese group was $91.71+$ 13.68 with a BMI $42.47+6.03$ obesity Vs $38.72+3.48$ (F value significant at 0.001 ).
Quality of Life in patients after bariatric surgery and in obese patients who have not undergone bariatric surgery.

Table 1: Distribution of subjects based on mean QoL in eight dimensions $\mathbf{N}=\mathbf{2 0 0}$

\begin{tabular}{|l|c|c|c|c|c|c|c|c|}
\hline \multirow{2}{*}{ Dimensions } & \multicolumn{4}{|c|}{ BS (n=100) } & \multicolumn{4}{c|}{ OBESE (n=100) } \\
\cline { 2 - 9 } & Min. & Max. & Mean & SD & Min. & Max. & Mean & SD \\
\hline Physical functioning (PF) & 15 & 100 & 86.95 & 23.41 & 0 & 95 & 52.5 & 22.78 \\
\hline $\begin{array}{l}\text { Role limitations due to } \\
\text { physical health (RPF) }\end{array}$ & 0 & 100 & 86.25 & 33.59 & 0 & 100 & 47.75 & 24.89 \\
\hline $\begin{array}{l}\text { Role limitation due to } \\
\text { emotional problems (REF) }\end{array}$ & 0 & 100 & 88.67 & 30.05 & 0 & 100 & 41.33 & 31.11 \\
\hline Energy fatigue (E) & 0 & 100 & 83.55 & 23.6 & 15 & 85 & 52.45 & 13.64 \\
\hline Emotional well being(EW) & 0 & 100 & 85 & 23.41 & 28 & 88 & 53.2 & 11.96 \\
\hline Social functioning (SF) & 0 & 100 & 83.88 & 30.01 & 13 & 100 & 55.5 & 17.13 \\
\hline Pain(P) & 0 & 100 & 77.85 & 28.27 & 10 & 100 & 60.75 & 24.2 \\
\hline General Health (GH) & 20 & 80 & 53.9 & 8.95 & 0 & 65 & 38.2 & 12.46 \\
\hline
\end{tabular}

Of the eight dimensions of QoL assessed, the mean scores in all the dimensions were found to be good in BS group with the highest score in Role Limitations due to Emotional Problems ie, REF $(88.67+30.05)$, followed by Physical Functioning ie, PF and Role Limitations due to Physical Health ie, RPF $(86.95+23.41,86.25+33.59$ respectively) and the lowest in General Health ie, GH $(53.90+8.95)$. On the other hand, the dimension $\mathrm{GH}$ was found to be very poor in obese group $(38.2+12.46)$ followed by REF with a score of $41.33+31.11$ and RPF $47.75+24.89$. The other dimensions showed only a slightly above average score.

Table 2: Distribution of subjects based on the physical and mental component mean scores of QoL $\mathbf{N}=\mathbf{2 0 0}$

\begin{tabular}{|l|c|c|c|c|c|c|c|c|}
\hline \multirow{2}{*}{ Component } & \multicolumn{4}{|c|}{ BS (n=100) } & \multicolumn{4}{c|}{ OBESE(n=100) } \\
\cline { 2 - 9 } & Min. & Max. & Mean & SD & Min. & Max. & Mean & SD \\
\hline Physical component score (PCS) & 5 & 100 & 83.68 & 21.8 & 3 & 84 & 52.34 & 18.95 \\
\hline Mental component score (MCS) & 0 & 100 & 85.74 & 22.72 & 25 & 83 & 49.98 & 11.84 \\
\hline
\end{tabular}

Both PCS and MCS were found to be high in BS group. The mental component score was higher $(85.74+$ 22.72) than the physical component score $(83.68+21.8)$ in BS group whereas the mental component score was poor in obese group $(49.98+11.84)$ than the physical component score $(52.34+18.95)$.
Table 3 Mean and Standard deviation of the overall QoL in BS group and obese group $\mathbf{N}=\mathbf{2 0 0}$

\begin{tabular}{|l|c|c|c|c|}
\hline QoL & Minimum & Maximum & Mean & SD \\
\hline QoL BS group & 22 & 94 & 80.93 & 18.18 \\
\hline QoL Obese group & 15 & 77 & 49.69 & 11.77 \\
\hline
\end{tabular}

The overall quality of life of BS group was found to be good (mean $80.93+18.18$ ) whereas it was poor in obese group $(49.69+11.77)$. 
Table 4: Distribution of the subjects based on their present general health compared to Oneyear ago. $\mathbf{N}=\mathbf{2 0 0}$

\begin{tabular}{|l|c|c|c|c|}
\hline \multirow{2}{*}{} & \multicolumn{2}{|c|}{ BS group (n=100) } & \multicolumn{2}{c|}{ Obese group (n=100) } \\
\cline { 2 - 5 } & f & $\mathbf{~ \% ~}$ & f & \% \\
\hline Much better & 62 & 62 & 25 & 25 \\
\hline Somewhat better & 15 & 15 & 25 & 25 \\
\hline Same & 8 & 8 & 42 & 42 \\
\hline Somewhat worse & 8 & 8 & 2 & 2 \\
\hline Much worse & 7 & 7 & & \\
\hline
\end{tabular}

Majority (62\%) in the BS group perceived their present general health much better compared to one year ago while only $6 \%$ of the obese group perceived it so. The rest $42 \%$ in the obese group perceived their general health as somewhat worse than one year ago and for $25 \%$, it was status quo.

Since the p-value obtained by the K-S test was less than 0.05 significance level in all the dimensions or components, the two groups were considered as heterogeneous. Hence Mann - Whitney U test was used to compare the mean scores of both the groups. It showed a highly significant statistical difference between the BS group and obese group in their Mean QoL (over all, dimension wise and component wise). Hence the null hypothesis was rejected.

Of the six socio-demographic variables (age, gender, marital status, education, occupation and type of work), statistically significant association was found between age, education, occupation and QoL ( $p$ value $<0.05$ ). A highly significant statistical association was found between duration after surgery and QoL in the BS group. $86 \%$ of the subjects studied were between 6 months to 5 years after LSG. Of, these $94.18 \%$ had good QoL. However, QoL was good only in nine out of fourteen subjects who had undergone bariatric surgery five years before.

\section{Discussion}

LSG is the recent bariatric surgery performed to reduce excess weight and improve QoL in morbidly obese. Though studies have shown that QoL can improve as soon as three months after bariatric surgery, the present study included only subjects who underwent LSG from six months to six years under the assumption that patients may have surgery related discomforts that may interfere in their QoL within six months. ${ }^{15}$
The mean scores of QoL (dimension wise,physical and mental componentsand overall) were found to be good in BS group than the obese group. Similar findings were observed in studies conducted mostly abroad. Substantial and significant improvement in physical and mental health favouring the surgical group was observed in a systematic review and meta-analysis on long term HRQL in bariatric surgery patients compared with controls spanning 5 to 25 years after surgery. ${ }^{16}$ Sleeve gastrectomy has been found to improve QoL and allows reduction of comorbidities. ${ }^{17,18}$

Studies by Akan et al., Fezzi et al., Porta et al. and Rebibo et al. demonstrated significant improvement in all domains of the SF-36 in 6 and 12 months after surgery compared to preoperative scores. ${ }^{1,20,21,22}$ Two studies in Indian settings also showed similar improvements in QoL one being in kerala. ${ }^{23,24}$

Long term studies like the famous Swedish Obese Subjects (SOS) intervention study, Norway(2014) systematic database search for a period of 10 years found significantly better outcome in the surgical group. But the peak improvements in HRQL after bariatric surgery was during the first year, whereas the weight regain phase (mainly between 1 and 6 year follow-up) was accompanied by a gradual decline in HRQL. ${ }^{25,26}$

Some studies could prove significant improvement only in selected dimensions of QoL.An Italian study (2014) to evaluate the changes of HRQL after bariatric surgery in 110 patients using SF- 36 showed a significant improvement in all dimensions of SF- 36 except General health and Mental health. ${ }^{27}$ In Poland the SF 36 scores were statistically different in all parameters $(\mathrm{P}<0.05)$ except for "role limitations attributed to emotional problems" and "mental health" in a group of 120 patients in comparison with patients scheduled for surgery. ${ }^{28} \mathrm{~A}$ 
systematic review with 72 studies on 9433 patients found that bariatric surgery has a significant positive influence on QoL in general with a greater positive influence on physical QoL compared to mental QoL. ${ }^{29,30,31,32,33}$

These findings are different from the present study findings in that the QoL had significantly improved in mental dimensions than the physical dimensions. The difference may be due to the improvement in self esteem, societal and family acceptance felt by the subjects probably influenced by the age, education and culture.

Contradictory to the findings of the present study, the physical and mental component scores were significantly found to be lower than the norm population mean in a Midwestern academic medical center study on 350 patients after bariatric surgery (2015). The study concluded that improvements in HRQL following bariatric surgery do not appear to be sustained over long term. ${ }^{34}$

Recent literature has raised concerns regarding the risk of adverse psychiatric events among bariatric surgery patients. The improvement in MCS score found in the present study is contrary to a recent study by Szmulewicz A, et al. 2019 who did a systematic review and meta-analysis of RCTs that compared surgical and non-surgical treatments and assessed mental health QoL of bariatric surgery patients. Eleven randomized trials with 731 participants were included in the finalanalysis. The final mentalhealth QoL scores were similar for surgically and non-surgically treated patients (SMD: $0.37,95 \%$ CI -0.07 to 0.81 ). These results suggest that intensive mental health follow-up following surgery should be routinely considered. ${ }^{35}$

In general it can be stated that most of the studies showed improvement in HRQL after LSG in 6-80 months follow-up in adult population. The impact varied considerably across studies showing a significantly greater positive influence on physical QoL compared to mental QoL.

Clinical Application: The compliance of the bariatric surgery subjects to the new lifestyle and changes during weight loss/weight regain periods could be enhanced only by consistent follow up by the community health workers resulting in better QoL. The fluctuations in weight loss after crossing certain threshold needs further longitudinal studies with multiple check points. ${ }^{36}$ Listening to the psychosocial issues of these patients would help in detecting early the manifestations of psychological disorders like depression so that appropriate referral services could be offered. Finally aggressive, comprehensive measures should be taken by the regional, state and national level governments to prevent obesity from childhood as patients after bariatric surgery have to live with restrictions hour as India ranks third in obesity in the world.

\section{Conclusion}

The study concludes that the health related quality of life is better in obese patients after LSG than those without surgery in the study subjects. Hence the null hypothesis is rejected. The study also strongly recommends the conduct of long term longitudinal qualitative studies with multiple points of measurements and follow-ups to be extended to the community.

Acknowledgement: The investigator deeply thanks the subjects for their active co-operation during the study.

\section{Conflict of Interest: Nil}

\section{Source of Funding: Self}

Ethical Clearance: The research proposal and tools were presented before the Institutional Scientific Committee and Institutional Ethical Committee and got approval. Permission for data collection was also obtained from the heads of the departments. Informed consent was obtained from each study subject

\section{References}

1. Alkassis M, Haddad FG, Gharios J, Noun R, Chakhtoura G. Quality of Life before and after Sleeve Gastrectomy in Lebanese Population. Journal of obesity. 2019;2019.

2. Hosseini SV, Al Hurry AM, Khazraei H, Hadavi H. The effect of laparoscopic sleeve gastrectomy on patients' quality of life in Shiraz. Journal of Minimally Invasive Surgical Sciences. 2018 Feb;7(1).

3. Nickel F, Schmidt L, Bruckner T, Buchler WB, Stich BP, Fischer L. Influence of bariatric surgery on quality of life, body image and general self-efficacy within 6 and 24 months- a prospective cohort study. Surgery for obesity and related diseases. [Internet].2016 month[date of citation];volume: [about 13 pages].

4. Brown D, Edwards H. Lewis's Medical Surgical 
Nursing- Assessment and management of clinical problems. $7^{\text {th }}$ ed. Place of publication: Elsevier; year. 2011 Page No. 976 - 992.

5. Le Foll D, Lechaux D, Rascle O, Cabagno G. Weight loss and quality of life after bariatric surgery: a 2-year longitudinal study. Surgery for Obesity and Related Diseases. 2020 Jan 1;16(1):56-64.

6. Still C, Sarwer DB, Blankenship J. The ASMBS Textbook of Bariatric Surgery. New York, NY: Springer Science, Media; 2014

7. Grothe KB, Dubbert PM, O'Jile JR. Psychological assessment and management of the weight loss surgery patient . American Journal of Medical Sciences, 2006, 331(4), 201- 206. doi: 00000441200604000-00006 [pii].

8. Song A, Fernstrom MH. (2008). Nutritional and psychological considerations after bariatric surgery. Aesthetic Surgery Journal, 28(2), 195199. doi: S1090-820X(08)00027- 7 [pii]10.1016/j. asj.2008.01.005

9. Moly KT, Shajan S, Johny S. Quality of Life and Depression in Obese. Indian Journal of Public Health Research \& Development. 2019;10(9):26670

10. Sarkhosh K, Birch DW, Sharma A, Karmali S. Complications associated with laparoscopic sleeve gastrectomy for morbid obesity: a surgeon's guide. Canadian journal of surgery. 2013 Oct;56(5):347.

11. Gilmartin J. Body image concerns amongst massive weight loss patients. Journal of clinical nursing. 2013 May;22(9-10):1299-309.

12. Sarwer DB, Wadden TA, Fabricatore AN. Psychosocial and behavioral aspects of bariatric surgery. Obesity research. 2005 Apr;13(4):639-48.

13. https://www.rand.org/health-care/surveys_tools/ mos/36-item-short-form.html

14. Sinha R, van den Heuvel WJ, Arokiasamy P. Validity and reliability of MOS short form health survey (SF-36) for use in India. Indian journal of community medicine: official publication of Indian Association of Preventive \& Social Medicine. 2013 Jan;38(1):22

15. Faria GF, Santos JM, Simonson DC. Quality of life after gastric sleeve and gastric bypass for morbid obesity. Porto Biomedical Journal. 2017 Mar 1;2(2):40-6. Quality-of-life measures improve as early as 3 months after bariatric surgery
16. Kristina F. Endocrinology Gastric Sleeve Works Long Term Obese patients., MedPage July 16, 2013, https://www.medpagetoday.org/endocrinology/ obesity/40490?vpass $=1$

17. Vasileios C, Georgios B, Vasileios L, Eelco de B. Quality-of-Life Before and After Laparoscopic Sleeve Gastrectomy. A Prospective Cohort Study. Article in Surgery for Obesity and Related Diseases 11(1) · May 2014 DOI: 10.1016/j. soard.2014.04.024

18. Flolo TN, Tell GS, Kolotkin RL, Aasprang A, Norekvål TM, Våge V, Hufthammer KO, Andersen JR. Changes in quality of life 5 years after sleeve gastrectomy: a prospective cohort study. BMJ open. 2019 Sep 1;9(9):e031170

19. Akan S, Uruc F, Aydin MT, Verit A. The effect of sleeve gastrectomy technique on women's sexual function: A prospective study. Rev Int Andrología [Internet]. 2018;16(4):167-73.

20. Fezzi M, Kolotkin RL, Nedelcu M, Jaussent A, Schaub R, Chauvet MA, et al. Improvement in Quality of Life After Laparo- scopic Sleeve Gastrectomy. Obes Surg [Internet]. 2011;21(8):1161-7.

21. Porta A, Aiolfi A, Musolino C, Antonini I, Zappa MA. Prospective Comparison and Quality of Life for Single-Incision and Conventional Laparoscopic Sleeve Gastrectomy in a Series of Morbidly Obese Patients. Obes Surg [Internet]. 2017;27(3):681-7.

22. Rebibo L, Verhaeghe P, Tasseel-Ponche S, Cosse C, Maréchal V, Dhahri A, et al. Does sleeve gastrectomy improve the gait parameters of obese patients? Surg ObesRelat Dis [Internet]. 2016;12(8):1474-81.

23. Bal RS, Ahuja A, Virk SS. Difference in Quality of Life in Morbidly Obese Patients after Bariatric Surgery. Poster [Internet]. 2012 .

24. Dasgupta A, Wasir J, Beloyartseva M, Malhotra S, Mithal A. An observational longitudinal study of the impact of sleeve gastrectomy on glycemic control in type 2 diabetes mellitus. Diabetes Technology and Therapeutics. 2013; 15(12): 990-995

25. Karlsson J, Taft C, Ryde A, Sjo S, Sullivan M. Ten -year trends in health related quality of life after surgical and conventional treatment for severe obesity; SOS intervention study. International Journal of Obesity.[Internet].2007 month[date of citation];31: [about 13 pages]. Available from www. 

nature.com site---- DOI:10.1038/SJ.IJO.0803573.

26. Andersen JR, Aasprang A, Karlsen TI, Natvig GK, Våge $V$, Kolotkin RL. Health-related quality of life after bariatric surgery: a systematic review of prospective long-term studies. Surgery for Obesity and Related Diseases. 2015 Mar 1;11(2):466-73.

27. Nadalini L, Zenti MG, Masotto L, Indelicato L, Fainelli G, Bonora F, Battistoni M, Romani B, Genna M, Zoppini G, Bonora E. Improved quality of life after bariatric surgery in morbidly obese patients. Interdisciplinary group of bariatric surgery of Verona (GICOV). Il Giornale di chirurgia. 2014 Jul;35(7-8):161.

28. Haldis, Lier, Eva Bringer, Oddbjorn Hove, BjarteStubhaug and Tong Tangen Health and Quality of life outcomes. Quality of life among patients undergoing bariatric surgery: associations with mental health. A 1 year follow-up study of bariatric surgery patients. http//www.hqlo.com/ content/9/1/79

29. Lindekilde N, Gladstone BP, Lübeck M, Nielsen J, Clausen L, Vach W, Jones A. The impact of bariatric surgery on quality of life: a systematic review and meta-analysis. Obesity reviews. 2015 Aug;16(8):639-51.

30. Hachem A, Brennan L. Quality of life outcomes of bariatric surgery: a systematic review. Obesity surgery. 2016 Feb 1;26(2):395-409.

31. Hosseini SV, Al Hurry AM, Khazraei H, Hadavi $\mathrm{H}$. The effect of laparoscopic sleeve gastrectomy on patients' quality of life in Shiraz. Journal of Minimally Invasive Surgical Sciences. 2018 Feb;7(1).

32. Van Nunen AM, Wouters EJ, Vingerhoets AJ, Hox JJ, Geenen R. The health-related quality of life of obese persons seeking or not seeking surgical or non-surgical treatment: a meta-analysis. Obesity surgery. 2007 Oct 1;17(10):1357

33. Bolen SD, Chang HY, Weiner JP, Richards TM, Shore $\mathrm{AD}$, et.al, Clinical outcomes after bariatric surgery: a five-year matched cohort analysis in seven US states. Obes Surg. 2012 May;22(5):74963. doi: 10.1007/s11695-012-0595-2

34. Khandalavala BN, Geske J, Nirmalraj M, KoranScholl JB, Neumann-Potash L, McBride CL. Predictors of health-related quality of life after bariatric surgery. Obesity surgery. 2015 Dec 1;25(12):2302-5.

35. Szmulewicz A, Wanis KN, Gripper A, Angriman F, Hawel J, Elnahas A, Alkhamesi NA, Schlachta CM. Mental health quality of life after bariatric surgery: A systematic review and meta-analysis of randomized clinical trials. Clinical obesity. 2019 Feb;9(1):e12290.

36. Prof. Moly K. T., "Excessive Weight Loss and Quality of Life after Laparoscopic Sleeve Gastrectomy-A Correlational Study", Indian Journal of Public Health Research \& Development, vol. 10 , no. 6 , pp. 296-301, 2019 